\title{
Single-nucleotide polymorphism array genotyping is equivalent to metaphase cytogenetics for diagnosis of Turner syndrome
}

\author{
Siddharth Prakash, MD, PhD¹, Dongchuan Guo, PhD¹, Cheryl L. Maslen, PhD², \\ Michael Silberbach, $\mathrm{MD}^{3}$, Dianna Milewicz, MD, PhD ${ }^{1}$, Carolyn A. Bondy, $\mathrm{MD}^{4}$ and the \\ GenTAC Investigators
}

\begin{abstract}
Purpose: Turner syndrome is a developmental disorder caused by partial or complete monosomy for the $\mathrm{X}$ chromosome in 1 in 2,500 females. We hypothesized that single-nucleotide polymorphism (SNP) array genotyping could provide superior resolution in comparison to metaphase karyotype analysis to facilitate genotypephenotype correlations.
\end{abstract}

Methods: We genotyped 187 Turner syndrome patients with 733,000 SNP marker arrays. All cases met diagnostic criteria for Turner syndrome based on karyotypes $(60 \%)$ or characteristic physical features. The SNP array results confirmed the diagnosis of Turner syndrome in $100 \%$ of cases.

Results: We identified a single X chromosome (45,X) in 113 cases. In 58 additional cases $(31 \%)$, other mosaic cell lines were present, including isochromosomes (16\%), rings (5\%), and Xp deletions (8\%).
The remaining cases were mosaic for monosomy $\mathrm{X}$ and normal male or female cell lines. Array-based models of X chromosome structure were compatible with karyotypes in 104 of 116 comparable cases (90\%). We found that the SNP array data did not detect X-autosome translocations (three cases) but did identify two derivative Y chromosomes and 13 large copy-number variants that were not detected by karyotyping.

Conclusion: Our study is the first systematic comparison between the two methods and supports the utility of SNP array genotyping to address clinical and research questions in Turner syndrome.

Genet Med advance online publication 6 June 2013

Key Words: array; cytogenetics; diagnosis; karyotype; sex chromosomes; Turner syndrome

culture. In some cases, lymphocytes have even been shown to accumulate chromosomal aberrations while in culture that are not detectable in whole blood. ${ }^{4,5}$ The likelihood that these artifacts will occur depends on the manner in which the samples are prepared. Cytogenetic analysis is a slow, labor-intensive, multistep process that is difficult to standardize and subject to considerable variability. As the cost declines and speed of analysis increases, whole-genome microarray methods have become the diagnostic standard for many chromosomal disorders but not for TS.

We hypothesized that single-nucleotide polymorphism (SNP) array genotyping could provide superior resolution in comparison to karyotyping to facilitate rapid diagnosis and genotype-phenotype correlations in TS. Here, we present the first systematic comparison between the two methods and provide evidence for the utility of SNP array genotyping to address clinical and research issues in TS.

\section{Subject selection}

\section{MATERIALS AND METHODS}

All women who were diagnosed with Turner syndrome (TS) based on suggestive phenotypic features were included in the study. For patients with peripheral blood-cell karyotypes, an 
$\mathrm{X}$ chromosomal abnormality was required to be present for inclusion. After reviewing the clinical data, five patients were excluded from further study because they did not meet diagnostic criteria for TS. These subjects had been labeled as "possible TS" and had not previously been genotyped. All other patients, including those with ring $\mathrm{X}$ chromosomes, had typical TS features and $\mathrm{X}$ chromosome abnormalities consistent with TS.

\section{Samples}

We studied 192 samples from females of European ancestry. We obtained 111 samples from the National Registry of Genetically Triggered Aortic Aneurysms and Other Cardiovascular Conditions (GenTAC, Rockville, MD). GenTAC is a consortium of eight institutions that is coordinated by the National Heart, Lung and Blood Institute, which maintains a central repository of tissue, DNA, and limited phenotypic data on patients with congenital heart disease, including TS. We also obtained 75 samples from the National Institute of Child Health and Human Development. An additional six samples were from the Baylor College of Medicine (two) and the University of Texas Health Science Center at Houston (four). Reports of karyotypes were available for 116 cases (60\%). All karyotypes were postnatal. Of 44 reports with mosaic cell lines, 27 reported 50-cell karyotypes and 9 reported karyotypes of 30 or fewer cells. The number of karyotyped cells was not reported in the other cases. Samples were collected simultaneously from the National Institutes of Health patients for karyotyping and genotyping. The relative timing of sample collections from GenTAC patients was not known to the investigators.

\section{Genotyping and copy-number analysis}

All DNA was obtained by extraction from whole blood. Sample processing, DNA purification, and hybridization were performed as previously described. ${ }^{6}$ Data for each Omni-Express BeadChip (Illumina, San Diego, CA) were normalized in GenomeStudio using information contained within the array. After allele detection and genotype calling, B-allele frequencies and $\log \mathrm{R}$ ratios were exported as text files for analysis with PennCNV software (http://www.openbioinformatics. org/penncnv). ${ }^{7}$ A second copy-number variant (CNV) detection algorithm, CNVPartition, was run as a plug-in within the GenomeStudio browser. Confidence thresholds and minimum number of probes per $\mathrm{CNV}$ were set to default values. Control genotypes from unrelated individuals of European ancestry in the database of Genotypes and Phenotypes were analyzed for copy-number variation using the same methods. ${ }^{8}$ DNA copy number and percentage of mosaicism were calculated using B-allele frequency and $\log \mathrm{R}$ ratio values for 18,239 SNPs along the length of the $\mathrm{X}$ chromosome. Nonmosaic $45, \mathrm{X}$ genotypes were identified by loss of heterozygosity across the entire $\mathrm{X}$ chromosome. B-allele frequency values were also used to determine the number of haplotypes present in isochromosome cell lines and map crossover events. Calculation of percentage of mosaicism for X and $\mathrm{Y}$ chromosome SNPs was based on the deviation of allele frequencies from expected values for copy losses or copy gains according to the method of Conlin et al. ${ }^{9}$ To deduce the most likely genotypes and estimate the percentages of mosaic cell lines, we compared mean B-allele frequency and $\log R$ ratio values of segmental aneuploidies with expected values for monosomies and trisomies. Figure 1 illustrates these computations for a TS case with a nonmosaic, isodicentric $\mathrm{X}$ chromosome. Array data and karyotypes were judged to be compatible if cell lines that were observed in more than one G-banded cell were also present in the genotypes.

\section{Statistical methods}

Chi-squared or Fisher's exact tests were applied to test quantitative parameters among the different study groups. All tests applied were two-tailed, and a $P$ value of $\leq 5 \%$ was considered statistically significant.

\section{Human subjects}

Studies were carried out with the approval of the Committee for the Protection of Human Subjects at the University of Texas Health Science Center at Houston, the GenTAC Scientific Advisory Committee (Rockville, MD), and the National Institute of Child Health and Human Development Institutional Review Board. All samples were from deidentified individuals who had been referred for clinical genetic testing. Karyotype information was provided by the treating physicians. After collection, all samples were deidentified to preserve patient confidentiality.

\section{RESULTS}

DNA samples from 187 TS patients (European American, with an average age of 32 years) were genotyped using 733,000 SNP marker arrays. One-hundred and sixteen cases had karyotypes, and 71 cases were thought to have TS based on characteristic physical features including short stature, premature ovarian failure, and congenital cardiovascular defects such as bicuspid aortic valve or coarctation (Table 1). Of note, genotyping confirmed the presence of X-chromosome aneuploidy consistent with the diagnosis of TS in all patients with a strong index of clinical suspicion for whom karyotypes were not available.

We identified a single $\mathrm{X}$ chromosome $(45, \mathrm{X})$ in 113 cases (60\%) and mosaic 45,X and 46,XX or 46,XY cell lines in 16 cases (9\%). In 58 additional cases (31\%), other structural variants were present, including isochromosomes (16\%), rings (5\%), and Xp deletions (8\%) (Table 1). Arrays did not detect the presence of three or more cell lines in any sample, whereas karyotyping identified a third cell line in a single case. Arrays did detect $Y$ chromosome material in 10 TS cases (5\%), including all 6 cases with Y-positive karyotypes and 2 cases that were not detected by karyotyping. This distribution of genotypes is not significantly different from that reported in the literature. ${ }^{10}$

Array-based models of X chromosome structure were compatible with karyotypes in 104 of 116 cases (90\%) with available data (see Supplementary Table S1 online). Sixty-three of these cases (55\%) had $45, \mathrm{X}$ genotypes, as compared with $60 \%(113 / 187)$ of all cases $(P>0.05)$. Array analysis showed that 19 of the $31 \mathrm{X}$ isochromosomes include proximal $\mathrm{Xp}$ 

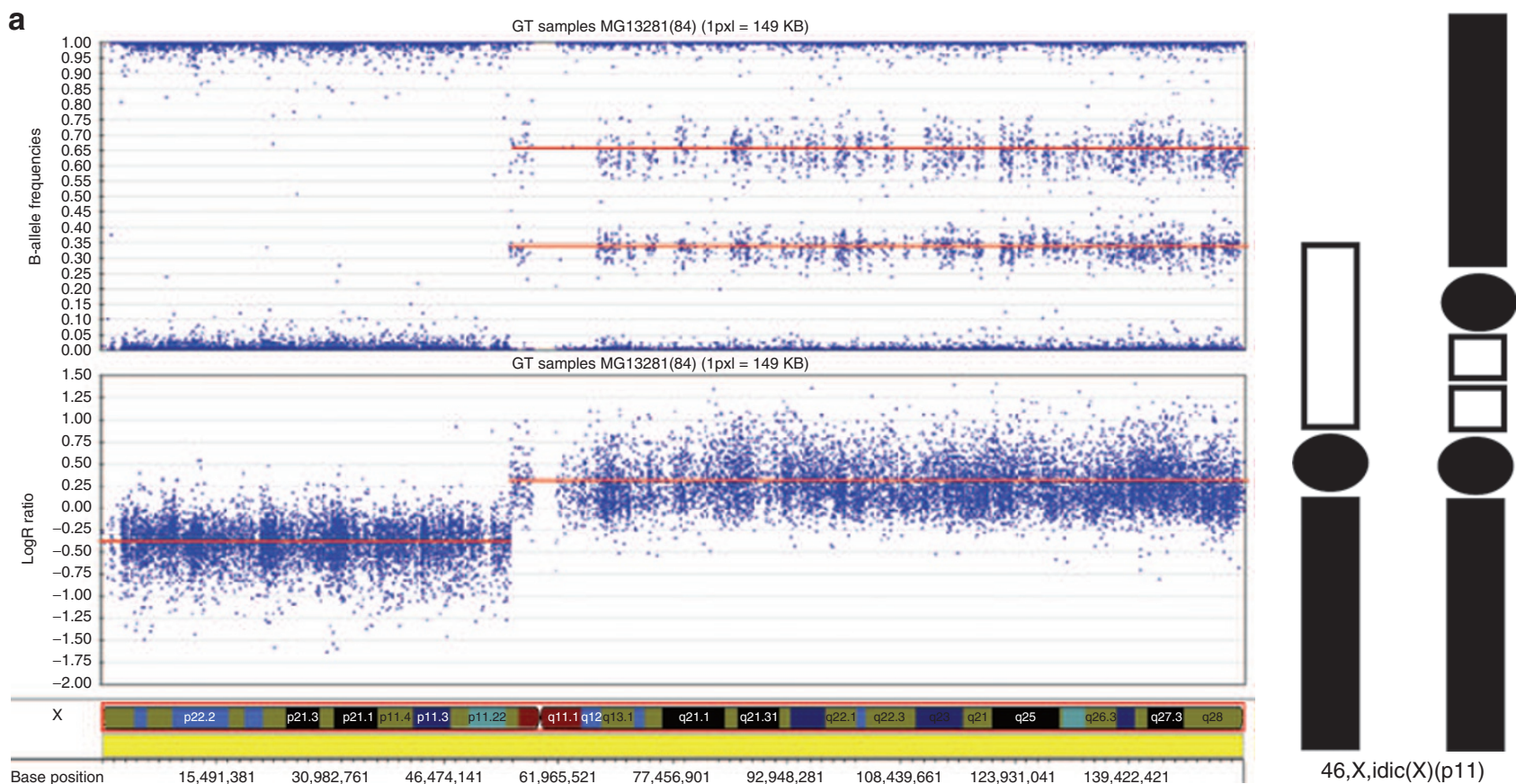

b
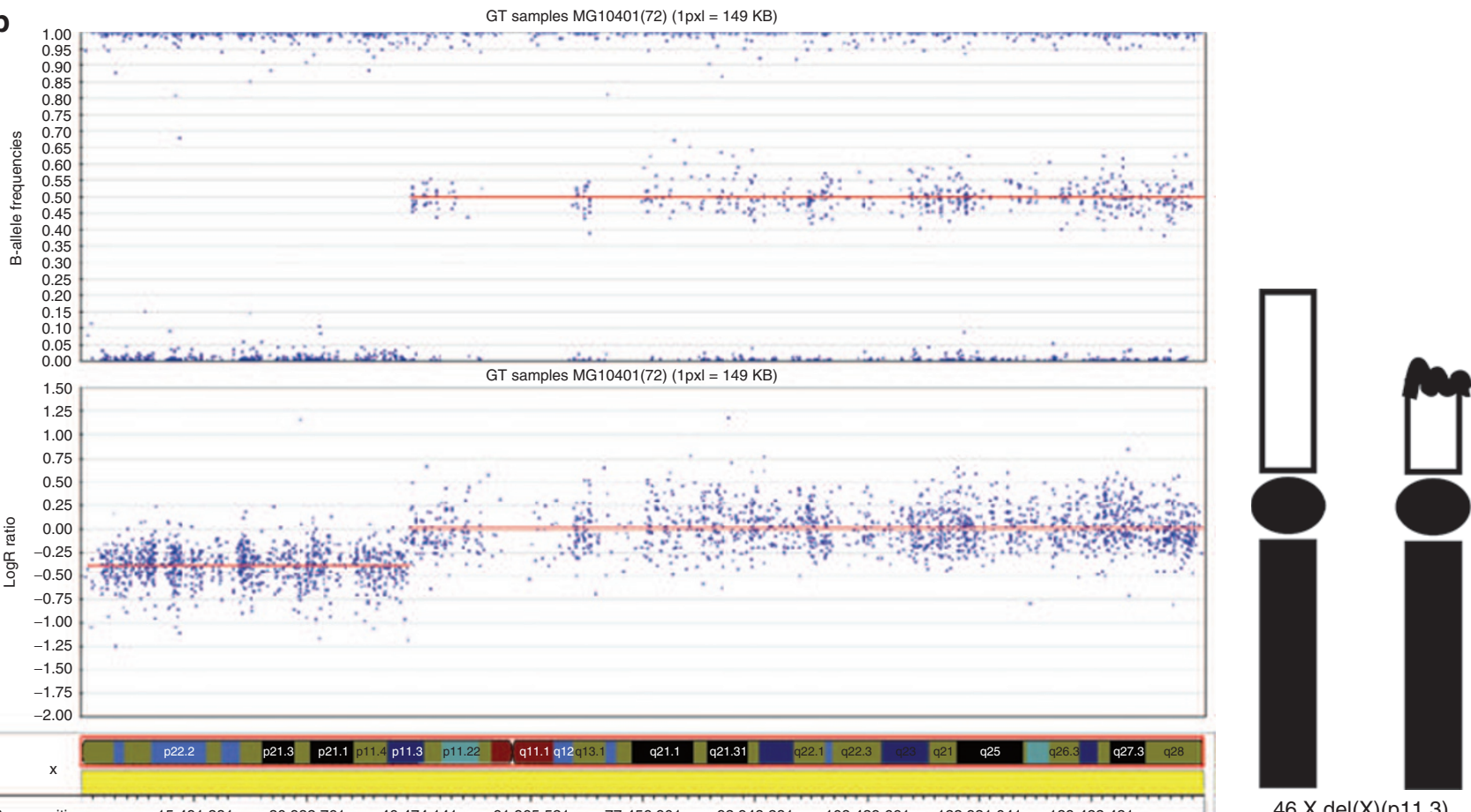

$46, X, \operatorname{del}(\mathrm{X})(\mathrm{p} 11.3)$

Figure 1 SNP array genotypes of two TS cases illustrate the computation of $\mathbf{X}$ chromosome structure. B-allele frequencies (BAFs) are plotted on the upper panel and corresponding LogR ratios (LRRs) on the lower panel. Mean values are indicated by red lines. Positive LRR values represent copy gains and negative LRR values represent copy losses. The X chromosome model corresponding to these values is illustrated on the right. Xp is white, $\mathrm{Xq}$ is black, and centromeres are oval. (a) A TS case with 46,X,idic(X)(p11). Homozygosity and mean LRR of -0.41 indicate segmental monosomy of Xpter-Xp11.22. The abrupt increase of LRR to 0.26 and division of BAF into four tracks $(1.00,0.66,0.34$, and 0$)$ indicate trisomy of Xp11-Xqter. (b) A TS case with $46, X$, del(X) (p11.3). Homozygosity and mean LRR of -0.39 indicate segmental monosomy of Xpter-Xp11.3. The abrupt increase of LRR to and division of BAF into three tracks $(1.00,0.50$, and 0$)$ indicate two copies of Xp11.3-Xqter. A mosaic such as 45,X[50]/46,X,i(X)(p11.3)[50] would appear similar to 46,X,del(X)(p11.3) in the array data but could be distinguished from the deletion by combined analysis of BAFs and LRRs. SNP, single-nucleotide polymorphism; TS, Turner syndrome. 
ORIGINAL RESEARCH ARTICLE

Table 1 Characteristics of 187 Turner syndrome cases

\begin{tabular}{lccc} 
& Mean & Quartile 1 & Quartile 3 \\
\hline Age (years) & 31.6 & 17.9 & 44.8 \\
\hline Height $(\mathrm{cm})$ & 145.2 & 140 & 152 \\
\hline Weight $(\mathrm{kg})$ & 58.9 & 47.3 & 69.6 \\
\hline BSA $\left(\mathrm{m}^{2}\right)$ & 1.52 & 1.37 & 1.69 \\
\hline & Number & Percentage & \\
\hline BAV & 68 & 35 & \\
Coarctation & 29 & 15 & \\
Genotype & & & \\
45,X & 113 & 60 & \\
45,X/46,X,iso $(X q)$ & 31 & 16 & \\
45,X/46,X,del $(X)$ & 16 & 8 & \\
$45, X / 46, X Y$ & 9 & 5 & \\
$45, X / 46, X, \operatorname{der}(X)$ & 8 & 4 & \\
$45, X / 46, X X$ & 7 & 4 & \\
$46, X, \operatorname{der}(X) t(X ; A)$ & 3 & 2 & \\
\hline
\end{tabular}

Values are presented as means and interquartile ranges, except for BAV and coarctation. There were no missing values for age, three missing values for height, and two missing values for weight. $\operatorname{Der}(\mathrm{X})$ indicates probable ring chromosome. Total of genotypes does not include translocations.

BAV, bicuspid aortic valve; BSA, body surface area.

material and are actually isodicentric. The breakpoints of these isochromosomes are clustered within a $5-\mathrm{Mb}$ region of Xp11 that is enriched for low-copy palindromic repeats (Figure 2). Recurrent Xp deletions and X-autosome translocations were also mapped to this interval. ${ }^{11,12}$ Our findings provide further evidence that $\mathrm{Xp} 11$ is uniquely susceptible to chromosomal rearrangements.

Eight of 12 cases that were discordant between the SNP and the karyotyped data involved mosaic cell lines that were detected in one but not both assays. Four cases with 45, X karyotypes were reclassified as mosaics according to SNP data, with a second cell line containing an abnormal sex chromosome or a normal cell line. Three cases had mosaic karyotypes but were found to be nonmosaic for monosomy X using SNP arrays. In five cases, arrays and karyotypes identified discrepant X chromosome abnormalities. In four of these cases, mosaicism was demonstrated in both arrays and karyotypes. These mosaic lines comprised between 4 and 30\% of sampled cells and included an intact $\mathrm{X}$ chromosome in one case and derivative chromosomes in seven cases (Table 2). In four of these eight cases, samples for arrays and karyotypes were known to have been collected simultaneously. We also found that the percentages of aneuploid cell lines as determined by arrays and karyotypes differed by more than twofold in 11 of 34 mosaic cases (32\%).

In four cases, the SNP data were compatible with two possible genotypes that result in identical array patterns: mosaics with equal 50\% contributions of 46,X,i(X) and 45,X cell lines, or nonmosaic Xp deletions (see Supplementary Table S1 online). To distinguish between these possibilities, we evaluated the chromosomal regions of the breakpoints, searched for
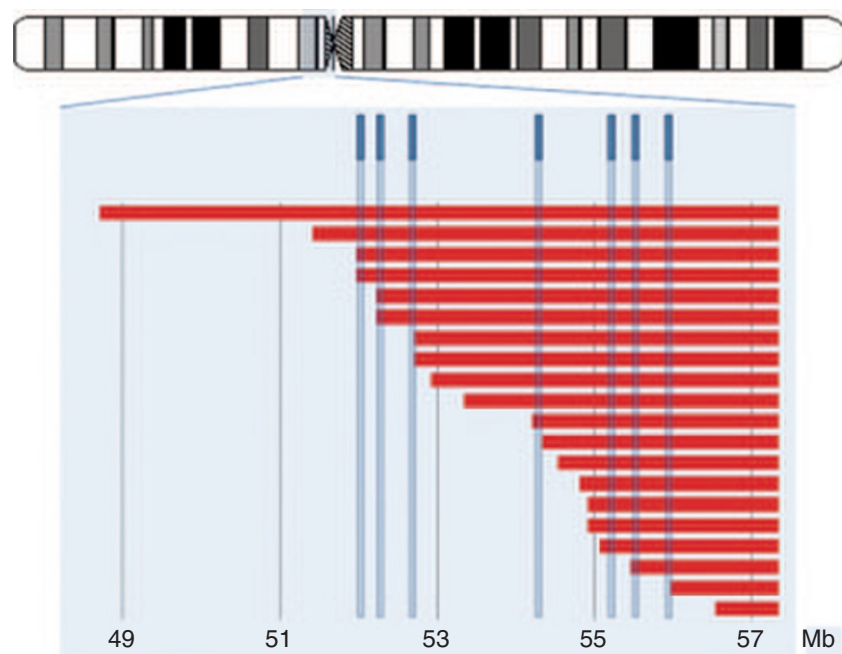

Figure 2 Location of 20 isochromosome breakpoints in Xp11.22-Xp11.1 in relation to an ideogram of the $X$ chromosome with the highlighted region and previously mapped low-copy-repeat sequences (blue rectangles). The breakpoints cluster in a region between 52 and $56 \mathrm{Mb}$ as previously shown by Koumbaris et al. (ref. 11). The array that was used in this study has an average maximum resolution of 8,000 base pairs.

Table 2 Discordant cytogenetic and microarray findings in 12 TS cases

\begin{tabular}{ll} 
Cyto & Array \\
\hline $45, X$ & $45, X[83 \%] / 46, X, \operatorname{der}(X)(p 22.12-q 27.3) 17 \%]$ \\
\hline $45, X$ & $45, X[75 \%] / 46, X, \operatorname{del}(Y)(q 11.21)[25 \%]$ \\
\hline $45, X$ & $45, X[85 \%] / 46, X, \operatorname{idic}(X)(p 11.22)[15 \%]$ \\
\hline $45, X$ & $45, X[83 \%] / 46 X X[17 \%]$ \\
\hline $45, X[80 \%] / 46, X, r(X)[20 \%]^{c}$ & $45, X$ \\
\hline $45, X[96 \%] / 46, X, r(X)[4 \%]^{b}$ & $45, X$ \\
\hline $45, X / 46, X, i s o(X q) / 47, X, i s o(X q), i s o(X q)^{c}$ & $45, X$ \\
\hline $46, X$, del$(X)(p 11.1)$ & $45, X$ \\
\hline $45, X[80 \%] / 46, X, i d i c(Y)(q 12)[20 \%]^{\text {a }}$ & $45, X[90 \%] / 46, X Y[10 \%]$ \\
\hline $45, X[88 \%] / 46, X, p s u i d i c(X)(q 21)[12 \%]^{b}$ & $45, X[97 \%] / 46, X, \operatorname{del}(X)(q 21.31)[3 \%]$ \\
\hline $45, X[98 \%] / 47, X X X[2 \%]^{b}$ & $45, X[98 \%] / 46, X X[2 \%]$ \\
\hline $45, X[85 \%] / 46, X X[15 \%]^{\text {a }}$ & $45, X[91 \%] / 46, X, \operatorname{del}(X)(q 21.1)[9 \%]$ \\
\hline
\end{tabular}

del, deleted chromosomes; der, derivative chromosomes, probably rings; idic, isodicentric chromosomes; iso, isochromosomes; SNP, single-nucleotide polymorphism; TS, Turner syndrome.

Clinical karyotypes (Cyto) were compared with the most likely karyotypes inferred from SNP array analysis (Array). The percentages of mosaic cell lines in the sample are bracketed. For Cyto cases, these were extrapolated from the number of karyotyped cells: ${ }^{a} 20,{ }^{b} 50$, or cunknown. Breakpoints are in parentheses.

additional haplotypes indicative of meiotic nondisjunction and precisely quantitated the $\log \mathrm{R}$ ratio deviations. Further analysis confirmed that the structural variants in all four of these cases were isochromosomes.

Array data successfully identified four marker chromosomes whose chromosomal origins were not identified in karyotypes as a $\mathrm{Y}$ chromosome, a derivative $\mathrm{Y}$ chromosome, a deleted $\mathrm{X}$ chromosome, and a ring X chromosome. SNP analysis of three TS cases with reported X-autosome translocations (2\%) showed 
Table 3 Large $\mathrm{X}$ chromosome CNVs detected by SNP arrays in 13 TS cases

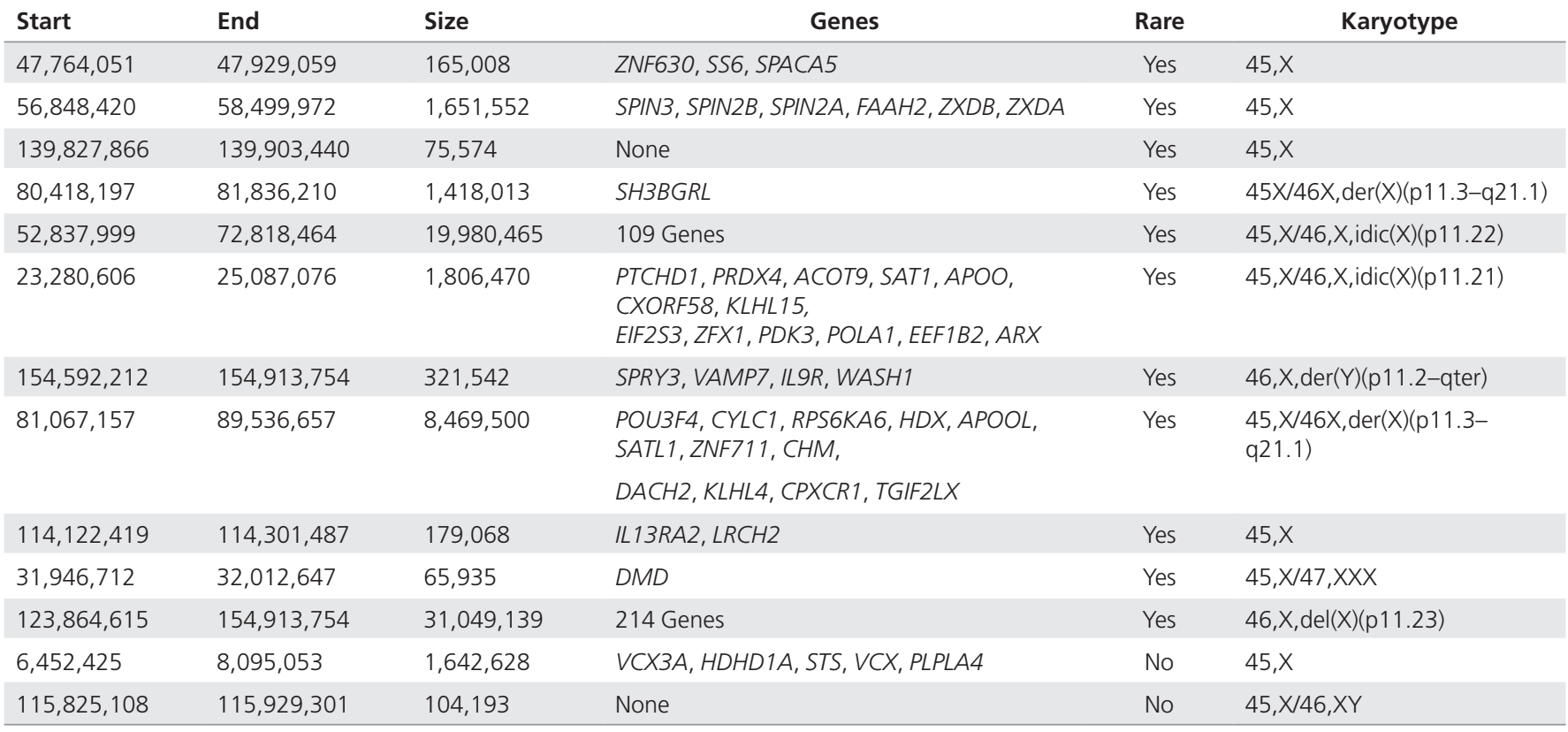

CNV, copy-number variant; SNP, single-nucleotide polymorphism; TS, Turner syndrome.

Start and end base-pair values are in hg18 coordinates. All CNVs were copy gains, except for 139,827,866-139,903,440, which was a copy loss. Genes: genes included in the CNV. Rare: yes if CNVs overlapped indicated regions in $<1 \%$ of 5,108 female controls from the database of Genotypes and Phenotypes. Karyotype: karyotype of individual with CNV.

that the translocations were unbalanced and resulted in copy losses with breakpoints in Xp. Two of these translocations did not cause autosomal copy changes. However, in the other case, unbalanced $t(X ; 9)$ resulted in trisomy $9 \mathrm{p}$. We were unable to determine whether typical features of the trisomy $9 \mathrm{p}$ syndrome (dysmorphic facial features and mental retardation) are present in this individual.

Arrays also identified 13 large X chromosome CNVs (CNVs $>100 \mathrm{~Kb}$ in size) in 12 different individuals that were not detected by karyotyping (Table 3). Five of these CNVs were extremely rare and were present in $<0.1 \%$ of controls. None of these CNVs involved genes with known roles in TS phenotypes.

\section{DISCUSSION}

Early diagnosis of TS is essential for interventions to restore normal or near-normal adult stature and for management of complications, including aortic coarctation, renal disease, or gonadoblastomas, which are prevalent in the subgroup with Y chromosome material. ${ }^{13}$ Four National Institutes of Health participants in this study underwent relevant evaluations, genetic counseling, and prophylactic gonadectomies according to the current standard of care after karyotypes disclosed cryptic $\mathrm{Y}$ chromosomes. ${ }^{14}$ TS patients without 45 , $\mathrm{X}$ genotypes tend to be diagnosed later in life but have similar rates of cardiovascular complications. ${ }^{15}$ Current PCR-based diagnostic methods have limited ability to detect the $40 \%$ of cases that involve mosaicism or X chromosome structural variants. ${ }^{16}$ Cytogenetic analysis by karyotyping is too labor intensive for rapid population-based screening and may not detect small fragments of Y chromosome material. Our data show that SNP array genotyping is a feasible alternative to karyotype testing for diagnosing TS. Moreover, the sensitivity and precision of SNP arrays may lead to improved genotype-phenotype correlations in TS.

TS may be caused by loss of an entire X chromosome $(45, \mathrm{X})$ in all cells, partial deletion of one X chromosome in all cells, or $\mathrm{X}$ chromosome deletions in a subset of cells (TS mosaicism). In almost all cases, the deletions cause partial or complete monosomy for Xp. Variation of Xq dosage may be extensive and includes duplications of $\mathrm{Xq}$ due to isochromosome formation in $15 \%$ of patients. SNP arrays were robustly able to detect all four categories of TS cases. We also showed that the SNP data led to the reclassification of four reported 45,X karyotypes (8\%) to mosaic TS cases and identified Y chromosome material that was not detected in two karyotypes. Ten of the large $\mathrm{X}$ chromosome CNVs found in 6\% of cases are rare or absent in controls and involve genes that may modify TS outcomes. It is important to note that rare CNVs were previously implicated in neurodevelopmental disorders and thoracic aortic aneurysms and dissections, both phenotypes that are relevant to TS. ${ }^{8,17}$ These data show that SNP arrays can provide additional prognostic value beyond karyotyping alone in the evaluation of TS patients.

Arrays and karyotypes were most frequently discordant due to differences in the detection of mosaic cell lines. In 4 of the 12 discordant cases, arrays led to incorrect interpretations of rare cell lines that were present in $<5 \%$ of sampled cells. This was most likely caused by technical limitations of the array data. However, six cases involved much larger variations in relatively abundant cell lines ( $>10 \%$ mosaicism) that 
could impact prognosis. We found no correlation between differences in the timing of sample collection and these discrepancies. Because both techniques are sensitive enough to detect mosaicism down to a level of $5 \%$, we propose that these larger differences most likely arose or were amplified during cell culture. ${ }^{9,18}$ Karyotypes are routinely produced after culturing peripheral blood lymphocytes for up to $72 \mathrm{~h}$. Under similar conditions, aneuploid cells have been shown to occur spontaneously in other human cell types, with the potential for multiple different mosaic lines to arise in consecutive cultures from the same individual. ${ }^{19-21}$ Aneuploid cells may also have a selective growth advantage or disadvantage compared with cocultured euploid cells from mosaic patients and may be amplified or suppressed by current culture methods. Moreover, in comparison with euploid cells, chromosomal instability appears to be more pronounced in TS patients. ${ }^{22-24}$ Mosaicism differences between karyotypes and arrays may also be accentuated due to sampling bias, especially if $<50$ cells are counted. SNP array analysis of whole blood eliminates the potential for selective pressure due to cell culture and may therefore provide a more accurate representation of mosaicism in peripheral blood.

One important limitation of this study is our inability to verify our findings using an independent method such as fluorescence in situ hybridization or by counting additional karyotyped cells. We found that array data lacks sufficient resolution to identify some low-abundance mosaic cell lines, which may be more accurately assessed in karyotypes. We also confirmed that karyotyping retains an important advantage over arrays to identify complex mosaicism, including translocations and rare $\mathrm{X}$ chromosome structural variants. SNP genotyping is unable to detect fully balanced $\mathrm{X}$-autosome translocations, but these were not present in our series.

In summary, the findings presented here indicate that SNP array analysis can be used to diagnose TS and may provide distinct advantages over karyotypes in the evaluation of TS.

\section{SUPPLEMENTARY MATERIAL}

Supplementary material is linked to the online version of the paper at http://www.nature.com/gim

\section{ACKNOWLEDGMENTS}

GenTAC participating centers and investigators are as follows: Johns Hopkins University, Kathryn W. Holmes, MD, Harry C. Dietz, MD, Williams Ravekes, MD, Kira Lurman, RN; University of Texas Health Science Center at Houston, Dianna M. Milewicz, MD, PhD, Meghan Terry, Alana Cecchi, MS, CGC; Baylor College of Medicine, Scott A. LeMaire, MD, Irina Volguina, PhD; Oregon Health \& Science University, Cheryl L. Maslen, PhD, Howard K. Song, MD, PhD, Victor Menashe, MD, Jessica D. Kushner, MS, CGC; University of Pennsylvania, Reed E. Pyeritz, MD, PhD, Joseph E. Bavaria, MD, Megan Morales; Weill Medical College of Cornell University, Craig T. Basson, MD, PhD, Richard Devereux, MD, Jonathan W. Weinsaft, MD, Deborah McDermott, MS, CGC; University of Michigan, Kim Eagle, MD; National Heart, Lung, and Blood Institute, H. Eser
Tolunay, PhD, Patrice Desvigne-Nickens, MD, Mario P. Stylianou, PhD, Megan Mitchell, MPH; RTI International, Barbara L. Kroner PhD, Donald Brambilla, PhD, Tabitha Hendershot, Danny Ringer, Meg Cunningham, Mark Kindem.

The authors are extremely grateful to patients for providing clinical data and samples for this study. The following sources provided funding to D.M.M. for these studies: RO1 HL62594 (D.M.M.), P50HL083794-01 (D.M.M.), Vivian L. Smith Foundation, Richard T. Pisani Funds, and TexGen Foundation. The National Institutes of Health UL1 RR024148 (CTSA) grant also provided funds for these studies. The GenTAC Registry has been supported by US federal government contracts HHSN268200648199C and HHSN268201000048C from the National Heart Lung and Blood Institute (Bethesda, MD) with RTI International (Research Triangle Park, NC). Additional support was provided by the National Institute of Arthritis and Musculoskeletal and Skin Diseases, the Oregon Clinical and Translational Research Institute (Portland, OR), grant UL1 RR024140 from the National Center for Research Resources (Bethesda, MD), and the Weill Cornell Medical College Clinical Translational Science Center (New York, NY), grant UL1RR024996.

\section{DISCLOSURE}

The authors declare no conflict of interest.

\section{REFERENCES}

1. Matura LA, Ho VB, Rosing DR, Bondy CA. Aortic dilatation and dissection in Turner syndrome. Circulation 2007;116:1663-1670.

2. Rao E, Weiss B, Fukami M, et al. Pseudoautosomal deletions encompassing a novel homeobox gene cause growth failure in idiopathic short stature and Turner syndrome. Nat Genet 1997;16:54-63.

3. Hirschfeldova K, Solc R, Baxova A, et al. SHOX gene defects and selected dysmorphic signs in patients of idiopathic short stature and Léri-Weill dyschondrosteosis. Gene 2012;491:123-127.

4. El-Zein R, Gu Y, Sierra MS, Spitz MR, Strom SS. Chromosomal instability in peripheral blood lymphocytes and risk of prostate cancer. Cancer Epidemiol Biomarkers Prev 2005; 14:748-752.

5. Salas C, Niembro A, Lozano V, et al. Persistent genomic instability in peripheral blood lymphocytes from Hodgkin lymphoma survivors. Environ Mol Mutagen 2012;53:271-280.

6. Peiffer DA, Le JM, Steemers FJ, et al. High-resolution genomic profiling of chromosomal aberrations using Infinium whole-genome genotyping. Genome Res 2006;16:1136-1148.

7. Wang K, Li M, Hadley D, et al. PennCNV: an integrated hidden Markov model designed for high-resolution copy number variation detection in whole-genome SNP genotyping data. Genome Res 2007;17:1665-1674.

8. Prakash SK, LeMaire SA, Guo DC, et al. Rare copy number variants disrupt genes regulating vascular smooth muscle cell adhesion and contractility in sporadic thoracic aortic aneurysms and dissections. Am J Hum Genet 2010;87:743-756.

9. Conlin LK, Thiel BD, Bonnemann CG, et al. Mechanisms of mosaicism, chimerism and uniparental disomy identified by single nucleotide polymorphism array analysis. Hum Mol Genet 2010;19:1263-1275.

10. Wolff DJ, Van Dyke DL, Powell CM; Working Group of the ACMG Laboratory Quality Assurance Committee. Laboratory guideline for Turner syndrome. Genet Med 2010;12:52-55.

11. Koumbaris G, Hatzisevastou-Loukidou H, Alexandrou A, et al. FoSTeS, MMBIR and NAHR at the human proximal Xp region and the mechanisms of human $\mathrm{Xq}$ isochromosome formation. Hum Mol Genet 2011;20:1925-1936.

12. Scott SA, Cohen N, Brandt T, Warburton PE, Edelmann L. Large inverted repeats within Xp11.2 are present at the breakpoints of isodicentric $X$ chromosomes in Turner syndrome. Hum Mol Genet 2010;19:3383-3393.

13. Wiktor A, Van Dyke DL. FISH analysis helps identify low-level mosaicism in Ullrich-Turner syndrome patients. Genet Med 2004;6:132-135. 
14. Bondy CA; Turner Syndrome Study Group. Care of girls and women with Turner syndrome: a guideline of the Turner Syndrome Study Group. J Clin Endocrinol Metab 2007;92:10-25

15. El-Mansoury M, Barrenäs ML, Bryman I, et al. Chromosomal mosaicism mitigates stigmata and cardiovascular risk factors in Turner syndrome. Clin Endocrinol (Oxf) 2007;66:744-751.

16. Rivkees SA, Hager K, Hosono S, et al. A highly sensitive, high-throughput assay for the detection of Turner syndrome. J Clin Endocrinol Metab 2011;96:699-705.

17. Pinto D, Pagnamenta AT, Klei L, et al. Functional impact of global rare copy number variation in autism spectrum disorders. Nature 2010;466: 368-372.

18. Hook EB. Exclusion of chromosomal mosaicism: tables of $90 \%, 95 \%$ and $99 \%$ confidence limits and comments on use. Am J Hum Genet 1977:29:94-97.

19. Ly P, Eskiocak U, Kim SB, et al. Characterization of aneuploid populations with trisomy 7 and 20 derived from diploid human colonic epithelial cells. Neoplasia 2011;13:348-357.
20. Liu AM, Qu WW, Liu X, Qu CK. Chromosomal instability in in vitro cultured mouse hematopoietic cells associated with oxidative stress. Am J Blood Res 2012;2:71-76.

21. Josse C, Schoemans R, Niessen NA, et al. Systematic chromosomal aberrations found in murine bone marrow-derived mesenchymal stem cells. Stem Cells Dev 2010;19:1167-1173.

22. Tyrkus M, Hoffman WH, Kraemer-Flynn KM. X chromosome instability associated with familial Turner syndrome. Clin Genet 1989;35: 111-115.

23. Park JP, Brothman AR, Butler MG, et al. Extensive analysis of mosaicism in a case of Turner syndrome: the experience of 287 cytogenetic laboratories. College of American Pathologists/American College of Medical Genetics Cytogenetics Resource Committee. Arch Pathol Lab Med 1999;123:381-385.

24. Sawyer JR, Swanson CM, Lukacs JL, et al. Telomeric fusion and chromosome instability in multiple tissues of a patient with mosaic Ullrich-Turner syndrome. Am J Med Genet 1997;69:383-387. 